\title{
Riqueza de la familia Orchidaceae en un bosque mesófilo de montaña en Chocamán, Veracruz, México
}

\section{Richness of the Orchidaceae family in a cloud forest of Chocamán, Veracruz, Mexico}

\author{
Olga Tejeda-Sartorius 1,3 (D, María de los Ángeles Aída Téllez-Velasco² (B)
}

1 Colegio de Postgraduados, Campus Montecillo, Carretera México-Texcoco km 36.5, Montecillo, 56230 Texcoco, Estado de México, México.

2 Universidad Nacional Autónoma de México, Jardín Botánico del Instituto de Biología, Delegación Coyoacán, 04510 Cd. Mx., México.

3 Autor para la correspondencia: olgats@colpos.mx

Citar como:

Tejeda-Sartorius, O. y M. A. Téllez-Velasco. 2017. Riqueza de la familia Orchidaceae en un bosque mesófilo de montaña en Chocamán, Veracruz, México. Acta Botanica Mexicana 121: 139-149. DOI: http://dx.doi.org/10.21829/abml21.2017.1177

Recibido: 26 de octubre de 2016

Revisado: 20 de abril de 2017.

Aceptado: 23 de junio de 2017.

DOI:

hetp://dx.doi.org/10.21829/abml21.2017.1177

\section{Resumen:}

Antecedentes y Objetivos: Existen fragmentos de bosque mesófilo de montaña (BMM) en el centro de Veracruz que no se han estudiado florísticamente. El objetivo de la presente investigación fue registrar la riqueza de orquídeas en un fragmento de este tipo de bosque en Chocamán, Veracruz.

Métodos: Se hizo trabajo de recolección de ejemplares, así como revisión de colecciones científicas e información bibliográfica.

Resultados clave: El listado incluyó 36 especies pertenecientes a 25 géneros. Epidendrum fue el género con mayor número de especies (7), seguido por Dichaea, Lycaste, Oncidium, Prosthechea y Stelis, con dos cada uno, el resto de los géneros estuvo representado por una. Cuatro especies son endémicas de México (Epidendrum longipetalum, Gongora galeata, Oncidium incurvum y Trichocentrum pachyphyllum), y dos están protegidas por la legislación mexicana (O. incurvum y Stanhopea oculata). Epidendrum magnoliae y Epidendrum cf. radioferens son nuevos registros para la flora de Veracruz.

Conclusiones: La orquideoflora aquí estudiada enfrenta problemas debido al cambio de uso de suelo y la extracción ilegal para comercio, por lo que urgen medidas para su conservación.

Palabras clave: bosque de niebla, endemismo, listado florístico, NOM-059, orquideoflora, región prioritaria de bosque mesófilo de montaña.

\section{ABSTRACT:}

Background and Aims: Some fragments of cloud forest (CF) in central Veracruz have not been studied floristically. The aim of the present investigation was to record the richness of orchids in a fragment of this type of forest in Chocamán, Veracruz.

Methods: Specimens were collected and scientific collections and bibliographic information were revised.

Key results: The list included 36 species, belonging to 25 genera. Epidendrum was the genus with more species (7), followed by Dichaea, Lycaste, Oncidium, Prosthechea and Stelis, with two each one, and all other genera were represented by one species. Four species are endemic to Mexico (Epidendrum longipetalum, Gongora galeata, Oncidium incurvum and Trichocentrum pachyphyllum); and two are protected by the Mexican legislation (O. incurvum and Stanhopea oculata). Epidendrum magnoliae and Epidendrum cf. radioferens are new records for the Veracruz flora.

Conclusions: The orchid flora studied here faces problems because of the land-use change and illegal extraction for trade, thus conservation measures are urgent.

Key words: cloud forest, endemism, floristic list, NOM-059, orchid flora, priority region of cloud forest. 


\section{INTRODUCCIÓN}

La familia Orchidaceae constituye uno de los grupos de plantas más vulnerables a los desafíos ambientales (Vi1laseñor, 2010), ya que son plantas asociadas a ambientes específicos, y pueden indicar la presencia o abundancia de otras especies (Newmark, 2002). Las orquídeas están adaptadas a factores abióticos del hábitat, tienen una relación simbiótica con las micorrizas y presentan mecanismos de polinización definidos (Akhalkatsi et al., 2014).

En México están registradas 1260 especies y 170 géneros de orquídeas, con $40 \%$ de endemismos (SotoArenas et al., 2007a; Salazar, 2009) y ocupan el tercer lugar a nivel nacional en cuanto a mayor diversidad taxonómica, siendo superadas por las familias Asteraceae y Fabaceae (Villaseñor, 2003; Hágsater et al., 2005).

Los ecosistemas de México más ricos y diversos en orquídeas son el bosque mesófilo de montaña (BMM), donde se encuentran $60 \%$ de sus especies, así como la selva tropical húmeda del sur del país (Rzedowski, 1996; Hágsater et al., 2005; Salazar, 2009).

A nivel mundial, el BMM es un ecosistema amenazado, ocupa un área de casi $381,000 \mathrm{~km}^{2}$, aproximadamente $0.26 \%$ de la superficie terrestre y representa $2.5 \%$ de la superficie total de los bosques tropicales (SánchezRamos y Dirzo, 2014). Se estima que en México ocupa cerca de $17,000 \mathrm{~km}^{2}$, lo que representa menos de $1 \%$ del territorio nacional (Sánchez-Ramos y Dirzo, 2014). Este ecosistema enfrenta serias amenazas que ponen en peligro su biodiversidad, tales como la ganadería, la tala ilegal y, en menor medida, aunque también con impactos severos, la conversión a cultivos agrícolas y la expansión de los asentamientos humanos (CONABIO, 2010). Otros autores señalan al cambio climático, el calentamiento global y la extracción selectiva de especies como importantes factores de riesgo para las orquídeas (Soto-Arenas et al., 2007b). El tipo de bosque mencionado en el estado de Veracruz cubre un área de 135,271 ha aproximadamente, incluyendo cafetales bajo sombra que se desarrollan en su cobertura (Castillo-Campos et al., 2011).

Después de Chiapas y Oaxaca, Veracruz es el estado mexicano con mayor biodiversidad (Gómez-Pompa y Castillo-Campos, 2010). Para Orchidaceae, uno de sus grupos más importantes, gracias al trabajo florístico realizado en el estado, se ha proporcionado continuamente material para describir nuevas especies o registrar taxones previamente no conocidos en su flora (Sosa y Gómez-Pompa, 1994; García-Cruz y Sánchez, 1999; Salazar, 2005; Castillo-Campos et al., 2009; Solano-Gómez, 2010; García-Cruz y Sosa, 2011; Castañeda-Zárate et al., 2012). Así, el registro más actual de orquídeas de Veracruz asciende a 433 especies (Castañeda-Zárate et al., 2012). La riqueza de Orchidaceae en el BMM de México es de 443 especies repartidas en 139 géneros (Villaseñor y Gual-Díaz, 2014), de las cuales en el BMM de Veracruz prosperan 257 especies en 93 géneros (Villaseñor, 2010).

Las orquídeas son un grupo botánico característico en la composición y estructura del BMM (Rzedowski, 1996; Williams-Linera et al., 2002; Castillo-Campos et al., 2005; García-Franco et al., 2008; García-Franco, 2012; Espejo-Serna, 2014; Villaseñor y Gual-Díaz, 2014). Varios trabajos incluyen exclusivamente a la familia Orchidaceae del BMM del centro de Veracruz (WilliamsLinera et al., 1995; García-Franco et al., 2008; TorresCantú, 2009; Viccon-Esquivel, 2009; Castillo-Hernández, 2013), pero los listados de florística local siguen siendo un complemento importante al inventario de orquídeas de dicho ecosistema en la región.

Ruíz-Jiménez et al. (2012) mencionan que en 18 localidades del centro de Veracruz se han llevado a cabo estudios florísticos o ecológicos en el BMM. Sin embargo, a pesar de las investigaciones realizadas, existen fragmentos aún sin explorarse (García-Franco et al., 2008) en los cuales la orquideoflora requiere ser analizada. Una de estas áreas se encuentra en el municipio Chocamán, donde las orquídeas son un componente importante de su biodiversidad. Aun cuando en dicha región se mantienen zonas conservadas, otras han sido deforestadas y destinadas a actividades agropecuarias tradicionales (PMD, 2008-2010), lo que pone en riesgo las poblaciones de orquídeas. En trabajos preliminares de la zona, TejedaSartorius et al. (2013) reportaron 34 especies, mientras que Baltazar-Bernal et al. (2014), 19. 
El objetivo del presente estudio fue complementar el inventario de Orchidaceae en un fragmento de BMM en el suroeste de Chocamán, Veracruz.

\section{Materiales y Métodos}

\section{Área de estudio}

El área de estudio se localiza al suroeste del municipio Chocamán, que pertenece a la zona denominada "Región de las Montañas", del estado de Veracruz, la cual comprende las comunidades de Tepexilota y algunos otros poblados más pequeños como El Carrizal y Guayabos. El sitio se ubica entre los márgenes de los ríos Metlac y Texalapa dentro de un polígono que presenta las siguientes coordenadas extremas: $18^{\circ} 59^{\prime} 40.1^{\prime \prime}-18^{\circ} 59^{\prime} 40.35^{\prime \prime} \mathrm{N}$, $97^{\circ} 05^{\prime} 54.17^{\prime \prime}-97^{\circ} 06^{\prime} 22.58^{\prime \prime O}$ y $18^{\circ} 58^{\prime} 23.18^{\prime \prime}-18^{\circ} 59^{\prime} 40.1^{\prime \prime N}$; $97^{\circ} 04^{\prime} 17.11^{\prime \prime}-97^{\circ} 05^{\prime} 54.17^{\prime \prime O}$. La altitud varía de 1322 a 1749 m (Fig. 1) (Baltazar-Bernal et al., 2014; SEFIPLAN, 2015).
El BMM en la zona de estudio presenta como especies arbóreas a Liquidambar styraciflua L. (ocozote), Platanus L. (álamo, haya), Quercus L. (encino), Fraxinus Tourn ex L. (fresno) y Populus L. (álamo).

\section{Revisión de herbarios}

Se revisaron ejemplares de la familia Orchidaceae provenientes del BMM en Veracruz y de manera particular, colectados en la región de estudio, depositados en las colecciones de los siguientes herbarios nacionales: AMO, CHAPA, CORU, FCMF, MEXU, XAL. Se consideraron ejemplares cuyas etiquetas indicaron "bosque mesófilo de montaña", "bosque de niebla", "bosque de neblina", "bosque de $L i$ quidambar" o "bosque de Pinus-Quercus con Liquidambar", según lo indicado por Villaseñor (2010). Se elaboró una base de datos en Microsoft Office Excel en donde se incluyeron los datos de las etiquetas de los ejemplares examinados de los herbarios indicados, así como los datos de los ejemplares colectados durante el presente estudio.

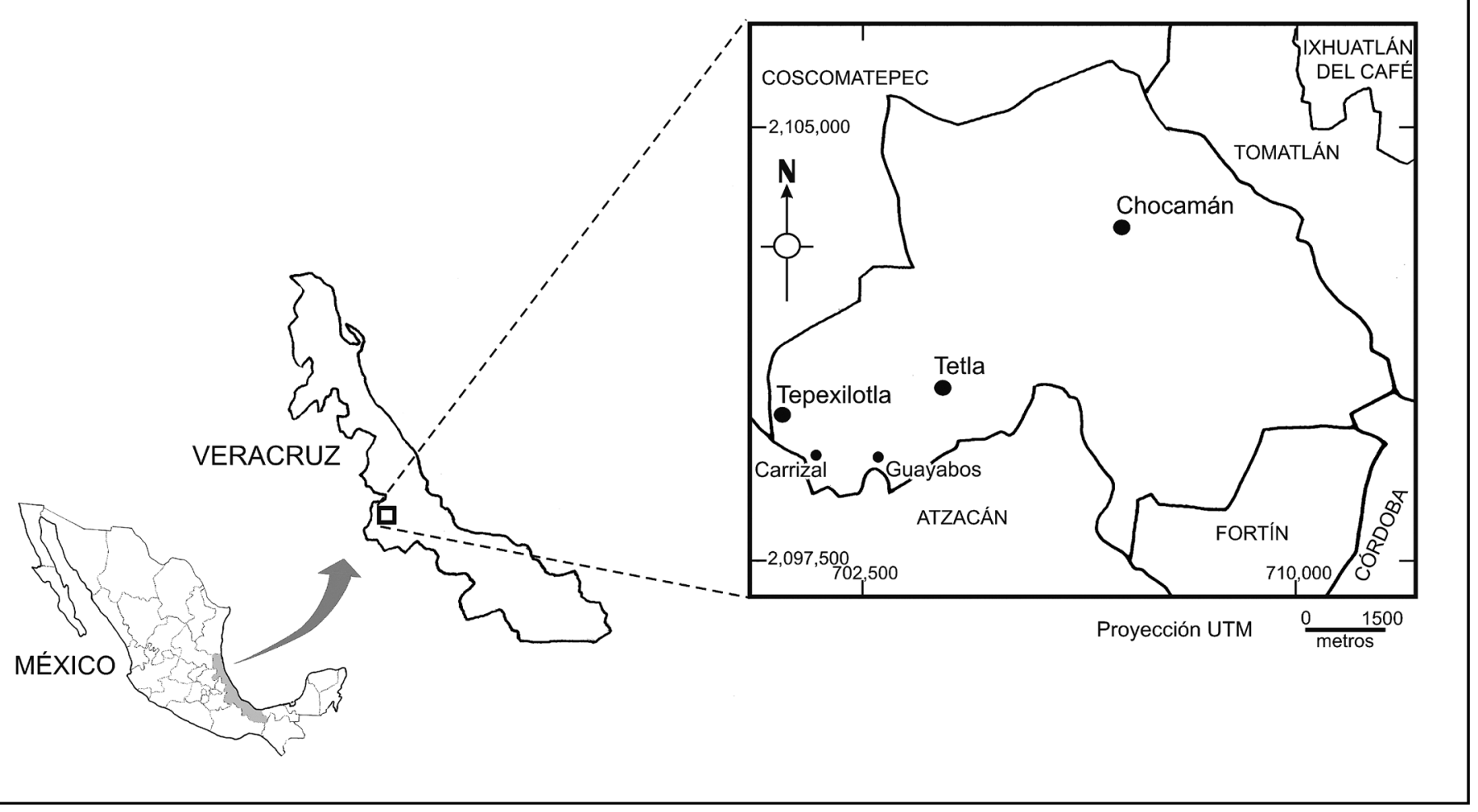

Figura 1: Ubicación de la zona de estudio, perteneciente al municipio Chocamán, Veracruz, México. 


\section{Recolección de orquídeas}

Los sitios de colecta fueron establecidos con ayuda de cartas topográficas i) Coscomatepec de Bravo E14B46, Puebla y Veracruz, Escala 1:50,000 y ii) Orizaba E14B56, Veracruz y Puebla, Escala 1:50,000 (INEGI, 2002); las cuales nos proporcionaron representatividad del área geográfica de estudio. Debido a lo abrupto de su topografía, se siguieron rutas de zonas conservadas indicadas por los pobladores locales. En total, se recorrieron $16.58 \mathrm{~km}$, cubriendo un área de $2.96 \mathrm{~km}^{2}$, donde se marcaron 200 puntos aleatorios de recolección; cada uno de éstos fue georreferenciado con un equipo GPS (GARMIN ${ }^{\circledR}$ eTrex Legend $\left.{ }^{\circledR} \mathrm{H}\right)$. Se realizaron 13 salidas de trabajo entre junio de 2012 y septiembre de 2013.

Los ejemplares se recolectaron en estado vegetativo, ya que los ejemplares en floración y/o fructificación fueron escasos. Para cada individuo observado se hizo un registro fotográfico y se colectó una división que fue trasladada al Jardín Botánico de la UNAM para su cultivo y resguardo, siguiendo las recomendaciones señaladas en el Plan de Manejo de la Colección (Téllez-Velasco, 2007). Cada semana se tomaron datos fenológicos de los ejemplares cultivados, y cuando éstos alcanzaron su estado de floración, se fotografiaron para hacer la determinación, así como la actualización de los nombres de las especies de acuerdo con trabajos taxonómicos recientes, tales como los de Pridgeon et al. (2005, 2009), Blanco et al. (2007), Soto-Arenas et al. (2007a) y Chase et al. (2015).

\section{Resultados}

\section{RIQUEZA DE ORQUÍDEAS}

La riqueza de orquídeas incluyó 36 especies pertenecientes a 25 géneros y dos subfamilias. Epidendrum L. fue el género con más especies (7), seguido por Dichaea Lindl., Lycaste Lindl., Oncidium Sw., Prosthechea Knowles \& Westc. y Stelis Sw., con dos cada uno. El resto de los géneros estuvo representado por una (Cuadro 1). En la figura 2 se observan algunas especies representativas del estudio.

La subfamilia Epidendroideae estuvo representada por 35 especies, una de las cuales (Govenia sp.) es terres- tre, hábito que comparte Stenorrhynchos cf. glicensteinii Christenson, de la subfamilia Orchidoideae.

Epidendrum cf. radioferens (Ames, F.T. Hubb. \& C. Schweinf.) Hágsater y Epidendrum magnoliae Muhl. se consideran nuevos registros para Veracruz. La primera solo es conocida de Oaxaca, Chiapas y Centroamérica; la segunda se conocía de Nuevo León, Tamaulipas y San Luis Potosí, así como del sureste de Estados Unidos de América.

En las colecciones herborizadas revisadas no se localizó ningún ejemplar proveniente de la zona específica de estudio, si bien hubo algunos registros depositados en AMO, CORU, FCMF, MEXU, XAL que indican "BMM" y "Chocamán" como lugar de procedencia: Epidendrum nitens Rchb.f., Isochilus major Schltdl. \& Cham., Prosthechea ochracea (Lindl.) W.E. Higgins, Prosthechea pseudopygmaea (Finet) W.E. Higgins, los cuales coincidieron con las especies encontradas en el presente trabajo, excepto E. nitens (Cuadro 1) la cual no se incluyó en nuestro listado porque la ficha de registro de la colección no contiene datos de latitud ni longitud que precise la localidad.

\section{Endemismo y categoría de riesgo de las especies inventariadas}

De las orquídeas presentes en el área de estudio cuatro especies son endémicas de México: i) Epidendrum longipetalum A. Rich. \& Galeotti, ii) Gongora galeata (Lind1.) Rchb.f., iii) Oncidium incurvum Barker ex Lindl., y iv) Trichocentrum pachyphyllum (Hook.) R. Jiménez \& Carnevali (Fig. 2, excepto la última). Dos especies están incluidas en la Norma Oficial Mexicana NOM-059-SEMARNAT-2010 (SEMARNAT, 2010) como especies amenazadas: Oncidium incurvum y Stanhopea oculata (G. Lodd.) Lindl. (Fig. 2).

\section{DISCUSIÓN}

En un trabajo preliminar, Tejeda-Sartorius et al. (2013) reportaron 34 especies incluidas en 24 géneros para la misma zona de estudio. Al finalizar el presente inventario, se modifica ese dato (36 especies y 25 géneros). Así, la riqueza de orquídeas reportadas para dicha área de estudio representa $8.12 \%$ de la orquideoflora nacional re- 
Cuadro 1: Especies presentes en el bosque mesófilo de montaña en la región suroeste de Chocamán, Veracruz, México. En=Endémica; NEn=No Endémica; A=Amenazada; Ep=Epífita; $\mathrm{T}=$ Terrestre. *=ejemplares observados in situ (solo se tienen fotografías digitales y no hay material en cultivo).

\begin{tabular}{|c|c|c|c|c|}
\hline Nombre científico & $\begin{array}{l}\text { Ejemplar de respaldo (registro fotográfico, } \\
\text { ejemplar cultivado o cita bibliográfica) }\end{array}$ & $\begin{array}{l}\text { Categoría de riesgo/ } \\
\text { Endemismo }\end{array}$ & $\begin{array}{l}\text { Hábito de } \\
\text { crecimiento }\end{array}$ & Altitud (m) \\
\hline Brassia verrucosa Bateman ex Lindl. & $\begin{array}{l}\text { Fotografía: IMG4875 - IMG4933* y } \\
\text { Baltazar et al., } 2014\end{array}$ & NEn & Ep & $\begin{array}{r}1430,1483 \\
1749\end{array}$ \\
\hline Camaridium densum (Lindl.) M.A. Blanco & MX-JB-008-DF-8112 & NEn & Ep & 1650 \\
\hline $\begin{array}{l}\text { Campylocentrum schiedei (Rchb.f.) Benth. ex } \\
\text { Hemsl. }\end{array}$ & Baltazar et al., 2014 & NEn & Ep & 1430 \\
\hline Coelia macrostachya Lindl. & $\begin{array}{l}\text { MX-JB-008-DF-8099 y Baltazar et al., } \\
2014\end{array}$ & NEn & Ep & $\begin{array}{r}1394,1438 \\
1681\end{array}$ \\
\hline Comparettia falcata Poepp. ex Endl. & Baltazar et al., 2014 & & Ep & 1394 \\
\hline Chysis leavis Lindl. & MX-JB-008-DF-8100 & NEn & Ep & 1505 \\
\hline Dichaea glauca (Sw.) Lindl. & MX-JB-008-DF-8097 & NEn & Ep & 1481 \\
\hline Dichaea neglecta Schltr. & MX-JB-008-DF-8098 & NEn & Ep & 1583 \\
\hline Elleanthus cynarocephalus (Rchb.f.) Rchb.f. & $\begin{array}{l}\text { MX-JB-008-DF-8101 y Baltazar et al., } \\
2014\end{array}$ & NEn & Ep & 1430,1649 \\
\hline $\begin{array}{l}\text { Epidendrum longipetalum A. Rich. \& } \\
\text { Galeotti }\end{array}$ & MX-JB-008-DF-8102 & En & Ep & 1667 \\
\hline Epidendrum magnoliae Muhl. & Baltazar et al., 2014 & NEn & Ep & 1430 \\
\hline Epidendrum melistagum Hágsater & Baltazar et al., 2014 & NEn & Ep & 1430,1322 \\
\hline Epidendrum mixtum Schltr. & MX-JB-008-DF-8103 & NEn & Ep & 1976 \\
\hline Epidendrum parkinsonianum Hook. & MX-JB-008-DF-8104 & NEn & Ep & 1710 \\
\hline $\begin{array}{l}\text { Epidendrum cf. radioferens (Ames, F.T. Hubb } \\
\text { \& C. Schweinf.) Hágsater }\end{array}$ & Fotografia: IMG3424 - IMG3959* & NEn & Ep & 1694 \\
\hline Epidendrum veroscriptum Hágsater & MX-JB-008-DF-8105 & NEn & Ep & 1384 \\
\hline Gongora galeata (Lindl.) Rchb. f. & $\begin{array}{l}\text { MX-JB-008-DF-8106 y Baltazar et al., } \\
2014\end{array}$ & En & Ep & $\begin{array}{r}1600,1409 \\
1358\end{array}$ \\
\hline Govenia sp. & Fotografia: IMG3284 - IMG3292* & NEn & $\mathrm{T}$ & 1527 \\
\hline Isochilus major Schltdl. \& Cham. & $\begin{array}{l}\text { MX-JB-008-DF-8107 y Baltazar et al., } \\
2014\end{array}$ & NEn & Ep & $\begin{array}{r}1322,1358 \\
1370,1394 \\
1483,1580\end{array}$ \\
\hline Jacquiniella equitantifolia (Ames) Dressler & MX-JB-008-DF-8108 & NEn & Ep & 1681 \\
\hline $\begin{array}{l}\text { Leochilus carinatus (Knowles \& Westc.) } \\
\text { Lindl. }\end{array}$ & $\begin{array}{l}\text { MX-JB-008-DF-8109 y Baltazar et al., } \\
2014\end{array}$ & NEn & Ep & $\begin{array}{c}1322,1358 \\
1370,1409 \\
1430,1467\end{array}$ \\
\hline Lycaste aromatica (Graham ex Hook.) Lindl. & $\begin{array}{l}\text { MX-JB-008-DF-8110 y Baltazar et al., } \\
2014\end{array}$ & NEn & Ep & 1483,1574 \\
\hline Lycaste deppei (Lodd.) Lindl. & $\begin{array}{l}\text { MX-JB-008-DF-8111 y Baltazar et al., } \\
2014\end{array}$ & NEn & Ep & 1483,1553 \\
\hline Malaxis wercklei (Schltr.) Ames & Fotografia: IMG3114 - IMG3127* & NEn & Ep & 1521 \\
\hline $\begin{array}{l}\text { Maxillariella variabilis (Bateman ex Lindl.) } \\
\text { M.A. Blanco \& Carnevali }\end{array}$ & MX-JB-008-DF-8113 & NEn & Ep & 1418 \\
\hline Oncidium incurvum Barker ex Lindl. & Fotografía: IMG3271* & $\mathrm{A} / \mathrm{En}$ & Ep & 1430 \\
\hline
\end{tabular}


Cuadro 1: Continuación.

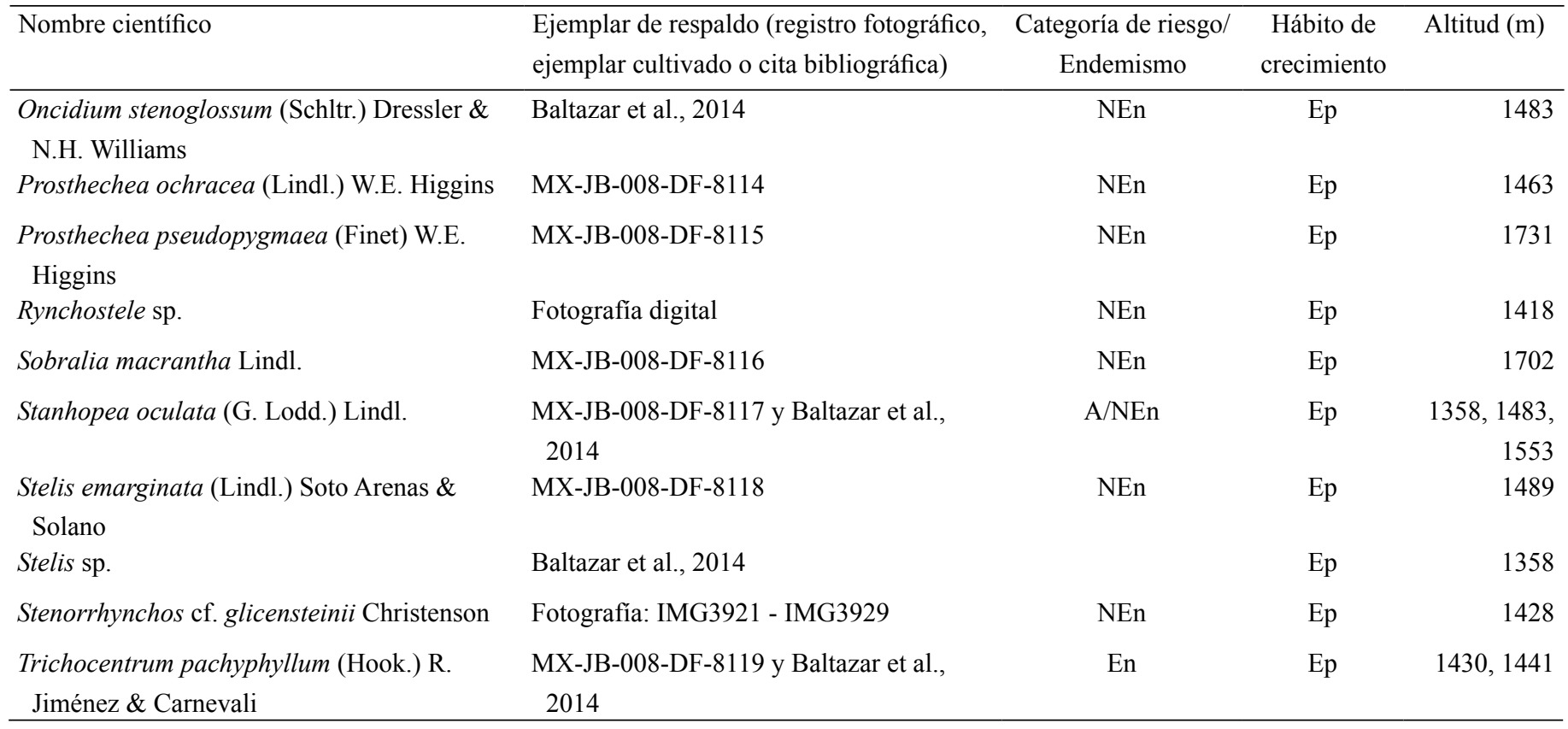

gistrada para el BMM de acuerdo con Villaseñor y GualDíaz (2014), así como 14\% de la que crece en el BMM de Veracruz, según Villaseñor (2010).

Baltazar-Bernal et al. (2014) reportaron 19 especies de orquídeas para una zona de Chocamán cercana a nuestros puntos de muestreo. Para fines exclusivos de una correcta comparación del listado de dichos autores con el que aquí se presenta, se consideró necesario actualizar los nombres científicos que fueron incorrectamente aplicados por dichos autores:

i) Dichaea pendula (Aubl.) Cogn., especie de las Antillas, Centro y Sudamérica, de la que no hay evidencia de su presencia en México (Llamacho y Larramendi, 2005). Por nuestras observaciones, consideramos que el nombre correcto es $D$. neglecta Schltr., la cual encontramos presente en la zona de estudio.

ii) Chysis aurea Lindl. no es nativa de México pero sí de Sudamérica. El nombre ha sido utilizado erróneamente para otras especies (Soto-Arenas et al., 2007a) y nosotros observamos in situ C. laevis Lindl. iii) Epidendrum paniculatum Ruiz \& Pav., se distribuye desde Belice hasta Argentina (Pridgeon, 1992; Dressler, 1993), por lo que se sugiere que el nombre correcto es E. veroscriptum Hágsater.

En relación con las especies Elleanthus cynarocephalus (Rchb.f.) Rchb.f., Epidendrum magnoliae y Trichocentrum pachyphyllum reportadas en el presente trabajo, Baltazar-Bernal et al. (2014) las mencionan con sus nombres sinónimos: Elleanthus capitatus (Poepp. \& Endl.) Rchb.f., Epidendrum conopseum R. Br. y Oncidium cavendishianum Bateman, respectivamente. Con base en lo anterior, se resume que de las 19 especies que Baltazar-Bernal et al. (2014) reportaron en su listado, 13 coincidieron con el nuestro. Las seis restantes se agregaron a nuestra investigación (Cuadro 1).

En su mayoría, las orquídeas de la zona de estudio son especies que se distribuyen en el BMM de otras regiones de México (Williams-Linera et al. 1995; PonceVargas et al., 2006; Torres-Cantú, 2009; Viccon-Esquivel, 2009; Pérez-Farrera et al., 2012; Castillo-Hernández, 



Figura 2: Especies de orquídeas presentes en el bosque mesófilo de montaña de la región suroeste de Chocamán, Veracruz. Especies endémicas de México: A. Epidendrum longipetalum A. Rich. \& Galeotti; B. Gongora galeata (Lindl.) Rchb.f.; especies incluidas en la NOM-059SEMARNAT-2010: C. Oncidium incurvum Barker ex Lindl.; D. Stanhopea oculata (G. Lodd.) Lindl. Otras especies que conformaron la riqueza de orquídeas de la zona de estudio: E. Epidendrum veroscriptum Hágsater; F. Lycaste aromatica (Graham ex Hook.) Lindl.; G. L. deppei (Lodd.) Lindl.; H. Prosthechea pseudopygmaea (Finet) W.E. Higgins; I. Dichaea neglecta Schltr.; J. Jacquiniella equitantifolia (Ames) Dressler; K. Sobralia macrantha Lindl.; L. Brassia verrucosa Bateman ex Lindl.; M. Coelia macrostachya Lindl.; N. Leochilus carinatus (Knowles \& Westc.) Lindl.; O. Chysis leavis Lindl.; P. Stenorrhynchos cf. glicensteinii Christenson; Q. Isochilus major Schltdl. \& Cham.; R. Maxillariella variabilis (Bateman ex Lindl.) M.A. Blanco \& Carnevali. 
2013) o bien, extienden su distribución hacia Centro y Sudamérica. Algunas de ellas también ocurren en las Antillas, como Dichaea glauca (Sw.) Lindl. y Elleanthus cynarocephalus. Tal como señala Rzedowski (1996), los géneros de orquídeas característicos del BMM de México son casi todos americanos.

Epidendrum mixtum Schltr., Epidendrum cf. radioferens, Malaxis wercklei (Schltr.) Ames y Stenorrhynchos. cf glicensteinii, presentes en la actual investigación, no habían sido reportadas previamente en otros trabajos (Williams-Linera et al., 1995; Torres-Cantú, 2009; Viccon-Esquivel, 2009; Castillo-Hernández, 2013) del BMM del centro de Veracruz.

De la riqueza de orquídeas de la zona de estudio, $11.1 \%$ son especies endémicas de México. Entre las aquí reportadas, Oncidium incurvum y Stanhopea oculata están consideradas en riesgo (Amenazadas) por la legislación mexicana, señaladas en la NOM-059-SEMARNAT-2010 (SEMARNAT, 2010). La conservación del área investigada enfrenta problemas debido al cambio de uso de suelo, convertida en potreros y tierras de cultivo, por lo que el peligro para las poblaciones de las orquídeas es alto. Acosta-Castellanos (2002) y Pérez-Farrera et al. (2012) señalaron que la familia Orchidaceae presenta el mayor número de taxa en riesgo en el BMM. Flores-Palacios y Valencia-Díaz (2007) documentaron que las orquídeas concentran el mayor número de especies comercializadas en un tianguis de Xalapa, Veracruz; 25 especies de nuestro inventario (69.4\%) son comercializadas ahí o en tianguis de flores de localidades cercanas a la zona de estudio (A. Téllez-Velasco y O. Tejeda-Sartorius, observación in situ), por lo que su extracción ilegal para el comercio local es una seria amenaza.

El BMM del presente estudio no está incluido dentro de las regiones prioritarias y/o subregiones con BMM de México (CONABIO, 2010), aunque podría ser parte de la región $\mathrm{V}$ denominada Centro de Veracruz, pues se encuentra muy cercana a las subregiones Huatusco-Coscomatepec y Orizaba. Se sugiere continuar analizando la orquideoflora de la zona investigada para atraer la atención de las autoridades correspondientes, para que se integre a los programas, acciones de conservación y manejo del BMM.

\section{CONCLUSIONES}

Para el área analizada se reporta una riqueza de 36 especies de orquídeas pertenecientes a 25 géneros. Cuatro son endémicas de México (Epidendrum longipetalum, Gongora galeata, Oncidium incurvum y Trichocentrum pachyphyllum), dos se encuentran en la categoría Amenazada (Oncidium incurvum y Stanhopea oculata). Epidendrum magnoliae y Epidendrum cf. radioferens son nuevos registros para Veracruz. Las principales amenazas de las orquídeas de la región de estudio son el cambio de uso de suelo y la extracción de ejemplares para el comercio ilegal.

Algunas medidas de conservación sugeridas son: i) destinar las áreas conservadas a pagos por servicios ambientales; ii) realizar proyectos de restauración forestal, de turismo rural, entre otros; iii) desarrollar protocolos de propagación y producción de orquídeas silvestres a través de la creación de Unidades de Manejo Ambiental (UMAS) y iv) destinar más recursos a la investigación, como a los listados florísticos y otros estudios ecológicos que señalen las especies en categoría de riesgo, principalmente.

\section{CONTRIBUCIÓN DE AUTORES}

OTS planeó y organizó el trabajo. OTS y MATV contribuyeron equitativamente en el desarrollo de la investigación: hicieron trabajo de campo y revisión de colecciones científicas, así como búsqueda bibliográfica y análisis de datos. MATV preparó material vegetal para jardín botánico. MATV y OTS realizaron determinación de las especies. OTS estructuró y escribió el manuscrito. MATV participó en la revisión, corrección, complementación y retroalimentación de cada sección del manuscrito. OTS y MATV elaboraron cuadro y figuras. Ambas autoras aprobaron el manuscrito.

\section{FINANCIAMIENTO}

El presente estudio fue financiado por la Línea Prioritaria de Investigación 4 y 13 (LPI 4 y LPI 13) del Colegio de Postgraduados. 


\section{AgRADECIMIENTOS}

Las autoras agradecen a la Dirección General de Vida Silvestre por la autorización de colecta científica de especies de la familia Orchidaceae en categoría de riesgo según la NOM-059-SEMARNAT-2010; así como a la Dirección General de Gestión Forestal y de Suelos, por la autorización para colecta de especies no enlistadas en la NOM059. Asimismo, se agradece al proyecto ORN-ORQ UNAM 2012-2013 de la Red de Orquídeas de SNICS-SINAREFI-SAGARPA. A Eder J. Guzmán Hernández por su apoyo en el trabajo de campo y a Gerardo Salazar por su apoyo en la determinación de algunas especies.

\section{LITERATURA CITADA}

Acosta-Castellanos, S. 2002. Plantas vasculares raras, amenazadas, o en peligro de extinción del estado de Oaxaca, un panorama preliminar. Polibotánica 13: 47-82.

Akhalkatsi, M., G. Arabuli y R. Lorenz. 2014. Orchids as indicator species of forest disturbances on limestone quarry in Georgia (South Caucasus). Journal Europäischer Orchideen 46(1): 123-160.

Baltazar-Bernal, O., J. Zavala-Ruiz, F. Y. Solís-Zanotelli, J. A. Pérez-Sato y O. Sánchez-Eugenio. 2014. Sendero interpretativo de orquídeas y bromelias en Tepexilotla, Chocamán, Veracruz. Revista Mexicana de Ciencias Agrícolas, Publicación especial 9: 1687-1699.

Blanco, M. A., G. Carnevali, W. M. Whitten, R. B. Singer, S. Koehler, N. H. Williams, I. Ojeda, K. M. Neubig y L. Endara. 2007. Generic realignments in Maxillariinae (Orchidaceae). Lankesteriana 7: 515-537.

Castañeda-Zárate, M., J. Viccon-Esquivel, S. E. RamosCastro y R. Solano-Gómez. 2012. Registros nuevos de Orchidaceae para Veracruz, México. Revista Mexicana de Biodiversidad 83(1): 281-284.

Castillo-Campos, G., M. E. Medina-Abreo, P. D. DávilaAranda y J. A. Zavala-Hurtado. 2005. Contribución al conocimiento del endemismo de la flora vascular en Veracruz, México. Acta Botanica Mexicana 73: 19-57. DOI: http://dx.doi.org/10.21829/abm73.2005.1004

Castillo-Campos, G., J. G. García-Franco, K. Mehltreter y M. L. Martínez. 2009. Registros nuevos de Ponthieva brenesii
(Orchidaceae) y Piper xanthostachyum (Piperaceae) para el estado de Veracruz, México. Revista Mexicana de Biodiversidad 80: 565-569.

Castillo-Campos, G., S. Avendaño-Reyes y M. E. MedinaAbreo. 2011. Flora y vegetación. In: Cruz-Angón, A. (ed.). La Biodiversidad en Veracruz: Estudio de Estado. Vol. I. Comisión Nacional para el Conocimiento y Uso de la Biodiversidad; Gobierno del Estado de Veracruz, Universidad Veracruzana, Instituto de Ecología, A.C. México, D.F., México. Pp. 161-179.

Castillo-Hernández, L. A. 2013. Inventario florístico del bosque mesófilo de montaña de la Reserva Bicentenario, Zongolica, Veracruz. Tesis de licenciatura. Facultad de Ciencias, Universidad Nacional Autónoma de México. México, D.F., México. 104 pp.

CONABIO. 2010. El Bosque Mesófilo de Montaña en México: Amenazas y Oportunidades para su Conservación y Manejo Sostenible. Comisión Nacional para el Conocimiento y Uso de la Biodiversidad. México, D.F., México. Pp. 80-85.

Chase, M. W., K. M. Cameron, J. V. Freudenstein, A. M. Pridgeon, G. Salazar, C. Van Den Berg y A. Schuiteman. 2015. An updated classification of Orchidaceae. Botanical Journal of the Linnean Society 177(2): 151-174. DOI: http://dx.doi.org/10.1111/ boj.12234

Dressler, R. L. 1993. Field guide to the orchids of Costa Rica y Panama. Cornell University Press. Ithaca, USA. Pp.70.

Espejo-Serna, A. 2014. Las plantas vasculares de los bosques mesófilos de montaña en México. In: Gual-Díaz, M. y A. Rendón-Correa (comps.). Bosques Mesófilos de Montaña de México: diversidad, ecología y manejo. Comisión Nacional para el Conocimiento y Uso de la Biodiversidad. México, D.F., México. Pp. 189-196.

Flores-Palacios, A. y S. Valencia-Díaz. 2007. Local illegal trade reveals unknown diversity and involves a high species richness of wild vascular epiphytes. Biological Conservation 136: 372-387. DOI: http://dx.doi. org/10.1016/j.biocon.2006.12.017

García-Cruz, J. y S. L. Sánchez. 1999. Orchidaceae II. Epidendrum. Flora de Veracruz 112: 1-110. 
García-Cruz, C. J. y V. Sosa. 2011. Las Orquídeas. In: Cruz Angón, A. (ed.). La Biodiversidad en Veracruz: Estudio de Estado. Vol. 2. Comisión Nacional para el Conocimiento y Uso de la Biodiversidad, Gobierno del Estado de Veracruz, Universidad Veracruzana, Instituto de Ecología, A.C. México, D.F., México. Pp. 191-199.

García-Franco, J. G. 2012. Epífitas: la otra parte del bosque. In: Williams-Linera, G. (ed.). El Bosque de niebla del centro de Veracruz: ecología, historia y destino en tiempos de fragmentación y cambio climático. Comisión Nacional para el Conocimiento y Uso de la Biodiversidad; Instituto de Ecología, A.C. Xalapa, México. Pp. 146-147.

García-Franco, J. G., G. Castillo-Campos, K. Mehltreter, M. L. Martínez y G. Vázquez. 2008. Composición florística de un bosque mesófilo del centro de Veracruz, México. Boletín de la Sociedad Botánica de México 83: 37-52.

Gómez-Pompa, A. y G. Castillo-Campos. 2010. La vegetación de Veracruz. In: Gómez-Pompa, A., T. Kroemer y R. Castro-Cortes (coords.). Atlas de la Flora de Veracruz: Un patrimonio Natural en Peligro. Secretaría de Educación de Veracruz; Gobierno del Estado de Veracruz. Tlalnepantla, México. Pp. 57-76.

Hágsater, E., M. A. Soto-Arenas, G. A. Salazar, M. R. Jiménez, M. A. López y R. L. Dressler. 2005. Las orquídeas de México. Instituto Chinoin, A.C. México, D.F., México. 302 pp.

INEGI. 2002. Cartas topográficas 1:50,000. Orizaba E14B56

y Coscomatepec de Bravo E14B46. Instituto Nacional de Estadística y Geografía, Geografía e Informática. Aguascalientes, México.

Llamacho, J. A y J. A. Larramendi. 2005. Las Orquídeas de Cuba. Greta Editores. Sevilla, España. Pp. 142-143.

Newmark, W. D. 2002. Conservation Biodiversity in East African Forest: a study of the Eastern Arc Mountains. Utah Museum of Natural History, University of Utah. Salt Lake City, USA. 191 pp.

Pérez-Farrera, M. A., R. Martínez-Camilo, N. MartínezMeléndez, O. Farrera-Sarmiento y S. Maza-Villalobos. 2012. Listado florístico del Cerro Quetzal (Polígono III) de la Reserva de la Biosfera El Triunfo, Chiapas, México. Botanical Sciences 90(2): 113-142.
PMD. 2008-2010. Plan Municipal de Desarrollo 2008-2010 del H. Ayuntamiento de Chocamán, Veracruz. Gaceta OficialGobierno del estado de Veracruz de Ignacio de la Llave. Tomo CLXXVIII, Núm. ext. 166, folio 687. Xalapa-Enríquez, Veracruz, México. 22 de mayo de 2008. Pp. 89-121.

Ponce-Vargas, A., I. Luna-Vega, O. Alcántara-Ayala y C. A. Ruiz-Jiménez. 2006. Florística del bosque mesófilo de montaña de Monte Grande, Lolotla, Hidalgo, México. Revista Mexicana de Biodiversidad 77: 177-190.

Pridgeon, A. M. 1992. The illustrated Encyclopedia of Orchids. Timber Press. Portland, USA. Pp. 127.

Pridgeon, A. M., P. J. Cribb, M. W. Chase y F. N. Rasmussen (eds.). 2005. Genera Orchidacearum. Epidendroideae Vol. 4 (part 1). Oxford University Press. New York, USA. Pp. 244-250.

Pridgeon, A. M., P. J. Cribb, M. W. Chase y F. N. Rasmussen (eds.). 2009. Genera Orchidacearum. Epidendroideae Vol. 5 (part 2). Oxford University Press. New York, USA. Pp. 131-135 y 174-176.

Ruiz-Jiménez, C. A., O. Téllez-Valdés e I. Luna-Vega. 2012. Clasificación de los Bosques Mesófilos de Montaña de México: afinidades de la flora. Revista Mexicana de Biodiversidad 83: 1110-1144.

Rzedowski J. 1996. Análisis preliminar de la flora vascular de los bosques mesófilos de montaña de México. Acta Botanica Mexicana 35: 25-44. DOI: http://dx.doi. org/10.21829/abm35.1996.955

Salazar, G. A. 2005. A new species of Ponthieva (Orchidaceae, Cranichidinae) from Veracruz, México. Brittonia 57(3): 252-254.

Salazar, G. A. 2009. Orquídeas. In: Lot, A., Z. Cano-Santana (eds.). Biodiversidad del Ecosistema del Pedregal de San Ángel. Universidad Nacional Autónoma de México. México, D.F., México. Pp. 153-169.

Sánchez-Ramos, G. y R. Dirzo. 2014. El Bosque Mesófilo de Montaña: Un Ecosistema Prioritario Amenazado. In: Gual-Díaz, M. y A. Rendón-Correa (comps.). Bosques Mesófilos de Montaña de México: Diversidad, Ecología y Manejo. Comisión Nacional para el Conocimiento y Uso de la Biodiversidad. México, D.F., México. Pp. 109-139. 
SEFIPLAN. 2015. Sistema de información municipal. Cuadernillos municipales, Chocamán. Secretaría de Finanzas y Planeación del estado de Veracruz. ceieg. veracruz.gob.mx/wp-content/uploads/sites/21/2016/05/ Chocamán1.pdf (consultado julio de 2014).

SEMARNAT. 2010. NORMA Oficial Mexicana NOM-059-SEMARNAT-2010. Protección ambiental-Especies nativas de México de flora y fauna silvestres-Categorías de riesgo y especificaciones para su inclusión, exclusión o cambioLista de especies en riesgo. Secretaría del Medio Ambiente y Recursos Naturales. Diario Oficial de la Federación. Cd. Mx., México. http://dof.gob.mx/nota_detalle.php?co digo $=5173091 \&$ fecha $=30 / 12 / 2010$

Solano-Gómez, R. 2010. Dos especies nuevas de Pleurothallidinae (Orchidaceae) de México. Lankesteriana 9(3): 447-454. DOI: http://dx.doi.org/10.15517/lank. v0i0.12106

Sosa, V. y A. Gómez-Pompa. 1994. Lista florística. Flora de Veracruz 82: 182-190.

Soto-Arenas, M. A., E. Hágsater, M. R. Jiménez, G. A. Salazar, G. R. Solano, G. R. Flores y R. I. Contreras. 2007a. Las orquídeas de México. Catálogo digital. Herbario AMO, Instituto Chinoin, A.C. México, D.F., México.

Soto-Arenas, M. A., R. Solano-Gómez y E. Hágsater. 2007b. Risk of extinction and patterns of diversity loss in Mexican orchids. Lankesteriana 7(1-2): 114-121.

Tejeda-Sartorius, O., M. A. A. Téllez-Velasco, E. J. GuzmánHernández. 2013. Las Orquídeas de Tepexilotla, Chocamán, Veracruz. Agroproductividad 6(3): 21-27.

Téllez-Velasco, M. A. A. 2007. Plan de Manejo de la Colección de Orquídeas del Jardín Botánico del Instituto de Biología, UNAM. Universidad Nacional Autónoma de México (UNAM)-Servicio Nacional de Inspección y Certificación de Semillas (SNICS)-Sistema Nacional de Recursos Fitogenéticos para la Alimentación y la Agricultura (SINAREFI)-Secretaria de Agricultura, Ganadería, Desarrollo Rural, Pesca y Alimentación (SAGARPA). México, D.F., México. 29 pp.
Torres-Cantú, G. B. 2009. Orquídeas (Epidendroideae y Vandoideae) presentes en los remanentes de vegetación original de la zona propuesta como reserva de la biósfera de las cuencas de los ríos Nautla, Copila y Misantla, Veracruz. Tesis de licenciatura. Facultad de Biología, Universidad Veracruzana. Xalapa, México. 381 pp.

Viccon-Esquivel, J. 2009. Riqueza y composición florística de las epífitas vasculares del bosque mesófilo de montaña de las localidades de Atzalán y Zongolica, Veracruz. Trabajo de experiencia recepcional. Facultad de Ciencias Biológicas y Agropecuarias, Universidad Veracruzana. Córdoba, Veracruz, México. 72 pp.

Villaseñor, J. L. 2003. Diversidad y distribución de las Magnoliophyta de México. Interciencia 28(3): 160-167.

Villaseñor, J. L. 2010. El Bosque Húmedo de Montaña en México y sus plantas vasculares: Catálogo FlorísticoTaxonómico. Comisión Nacional para el Conocimiento y Uso de la Biodiversidad; Universidad Nacional Autónoma de México. México, D.F., México. 38 pp.

Villaseñor, J. L. y M. Gual-Díaz. 2014. El Bosque Mesófilo de Montaña en México y sus plantas con flores. In: Gual-Díaz, M. y A. Rendón-Correa (comps.). Bosques Mesófilos de Montaña de México: Diversidad, Ecología y Manejo. Comisión Nacional para el Conocimiento y Uso de la Biodiversidad. México, D.F., México. Pp. 221-236.

Williams-Linera, G., V. Sosa y T. Platas. 1995. The fate of epiphytic orchids after fragmentation of a Mexican cloud forest. Selbyana 16(1): 36-40.

Williams-Linera, G., H. R. Manson y E. Isunza-Vera. 2002. La fragmentación del bosque mesófilo de montaña y patrones de uso del suelo en la región oeste de Xalapa, Veracruz, México. Madera y Bosques 8(1): 73-89. DOI: http://dx.doi.org/10.21829/myb.2002.811307 